\title{
MEASUREMENT OF CHANGES IN TISSUE METABOLISM USING A CLARK-TYPE OXYGEN SENSOR
}

\author{
Benjamin Franc**, Nathalia Peixoto*, Laurent Giovangrandi*, \\ Glen McLaughlin*, and Gregory T.A. Kovacs* \\ Department of Electrical Engineering* and Department of Nuclear Medicine** \\ Stanford University, Stanford, CA 94305-4075
}

\begin{abstract}
This paper presents data characterizing the ability to measure metabolic activity at a tissue interface using a Clark-type oxygen concentration sensor consisting of a microfabricated thinfilm electrode matrix overlaid with a solid-state proton conductive matrix and encapsulated in a bio-inert polytetrafluoroethylene film. HL-1 atrial myocytes were cultured onto the sensor surface. Cyclic voltammetry was utilized to characterize the sensor response over 30 minutes while the overlying tissue was subjected to either triiodothyronine or verapamil, drugs known to upregulate or downregulate, respectively, the metabolic activity of myocardial cells. Levels of percent of baseline current were calculated for both drugs, as well as for controls. The change of measured oxygen concentration was considered inversely proportional to the change in metabolic activity. Each of the drugs exerted a significant metabolic effect on the tissue, as evidenced by changes in the sensor current, thus confirming the ability to measure cellular metabolic changes at the tissue surface using the Clark-type oxygen sensor. Verapamil at $25 \mu \mathrm{g} / \mathrm{L}$ was found to increase oxygen concentration to $135 \pm 4 \%$ as compared to control values $(95 \pm 8 \%)$. Triiodothyronine at $2.5 \mu \mathrm{g} / \mathrm{L}$, on the other hand, decreased oxygen levels in the extracellular space to $85 \pm 9 \%$, as compared to baseline oxygen $(98 \pm 6 \%)$. The recorded metabolic effects of the tested drugs were consistent with their known physiologic effects. This sensor has possible applications in drug screening or toxicological detection.
\end{abstract}

\section{INTRODUCTION}

During the last few years, miniaturization has enabled more accurate modeling of drug effects on human tissues by means of in vitro testing with cultured tissue and has decreased the need for large populations of animal experiments. A large proportion of drug screening is based on binding affinity of the drug to a receptor of interest. However, much can be gained from knowledge of the actual physiologic effect of the drug on the cell of interest, especially in the case of screening metabolic depressants for organ preservation, anti-metabolites for the immune system, and chemotherapeutics.

Similarly, toxicological testing no longer requires extensive sample transport and processing times. Cell-based biosensors have demonstrated their usefulness in toxicity monitoring in the field and a cell-based biosensor system utilizing extracellular electrical recording from chick cardiac myocytes has been demonstrated [1]. More elaborate systems [2] to better classify analytes and general purpose hardware and software platforms also based on extracellular recordings have made the emergence of biosensors as toxicological analyzers in the field imminent [3]. However, these applications could benefit from the measurement of effects on cell physiology instead of those on electrical activity alone.

Characterization of cell physiology can be achieved by multiple means. The utilization of oxygen by the tissue is a function of cellular metabolism and the use of an oxygen sensor as a means of monitoring drug effects on cellular metabolism in vitro has been previously demonstrated by optical techniques. For example, an oxygen sensor utilizing fluorescence quenching has been used to monitor cellular metabolic response of tissue cultivated in a hollow fiber bioreactor to various drugs and toxins. Changes in oxygen concentration in the space adjacent to the cell were demonstrated to be a direct indication of metabolic demands of the cell. Drawbacks to this approach have included severe temperature sensitivity and the time required to attain stability [4]. In addition to these problems, oxygen sensors used in the medical and biotechnology fields have not yet been miniaturized for metabolic drug and toxicity screening. The size, inconsistent performance and short shelf life limit the utility of today's aqueous-based oxygen sensors [5].

In order to show the ability to detect toxic or metabolically active substances utilizing a microfabricated Clark-type oxygen sensor, a highly metabolically active cell line was sought. Cells utilized on such a metabolic sensor do not need to be electrically active, thus any type of robust, anchorage-dependent and metabolically active tissue may be used. Myocardium has very high oxygen demands; in the human body, resting myocardial tissue may extract as much as $75 \%$ of the oxygen from the arterial circulation whereas other tissues extract only 20-25\% [6]. Furthermore, an easily cultured cell line exists, the HL-1 from the AT-1 mouse atrial cardiomyocyte tumor lineage, and is capable of maintaining its contractile, morphological, and electrophysiological characteristics when cultured [7].

In addition to high oxygen utilization of the myocardium, there are many well-characterized drugs that affect myocyte metabolism, making it an ideal candidate tissue for a metabolic sensor. In particular, triiodothyronine (thyroid hormone, T3) is known to regulate oxygen consumption and oxidative phosphorylation, and has known inotropic and chronotropic effects. Nongenomic-based effects of T3 on cardiac tissue occur rapidly; within four minutes of cardiac myocyte exposure to T3, slow-inactivating sodium channels are recruited and calcium movement across the cell membrane is stimulated, resulting in more rapid and sustained contractions $[\mathbf{8 , 9}]$.

Verapamil, a calcium channel blocker, has the opposite effect on myocardial tissue metabolism as thyroid hormone. Verapamil acts at specific binding sites associated with potentialdependent calcium channels and thereby interferes with the slow inward calcium current, preventing the trigger current for myocardial contraction [6]. This, in turn, decreases the work done by the heart and the metabolic demand. Clinical studies have demonstrated electrophysiologic effects of verapamil within 1-2 minutes, with peak effect seen at 10 minutes and residual effects observed up to 6 hours following intravenous administration [10].

\section{METHODS}

Clark-type integrated oxygen sensors were fabricated as previously described [11]. Briefly, they consist of a micro-

Travel support has been generously provided by the Transducers Research Foundation and by the DARPA MEMS and DARPA BioFlips programs. 
fabricated thin-film electrode matrix overlaid with a solid-state proton conductive matrix (NafionTM:PVP-360) and coated with a polytetrafluoroethylene (PTFE) film, as is shown schematically in Figure 1. Fabrication of the electrode array was followed by integration into a standard dual in-line package. A cell culture dish with a $1 \mathrm{~cm}$ hole in the bottom was mounted over the chip, such that the surface of the sensor formed part of the floor of the culture dish. Inlet and outlet ports were provided through the top of the culture dish to allow physiological medium to flow through the chamber. Perfusion was maintained at $1 \mathrm{~mL} / \mathrm{min}$ by a peristaltic pump.

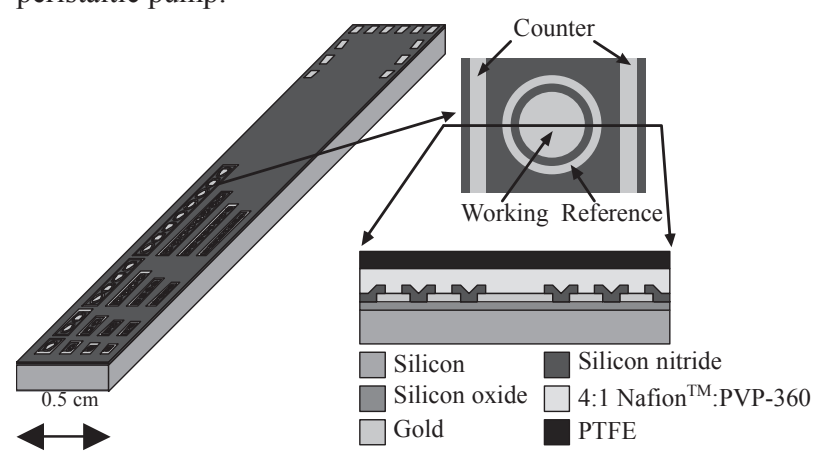

Figure 1. Single element cross-section of the dissolved gas sensor test matrix [11].

The oxygen arrays used for the experiments had either four elements with a working electrode diameter of $80 \mu \mathrm{m}$ or eight elements with working electrode diameter of $40 \mu \mathrm{m}$. An example of a fabricated sensor is shown in Figure 2.

A calibration curve was taken for each of the oxygen sensors by plotting the current obtained at the diffusion-controlled voltage against the oxygen concentration measured utilizing a standard handheld dissolved oxygen monitor (Oakton pH/DO 300 series, Oakton Instruments, Vernon Hills, Illinois), plotting it over three points, and curve fitting with a linear regression model. Linearity had been previously demonstrated in [11].

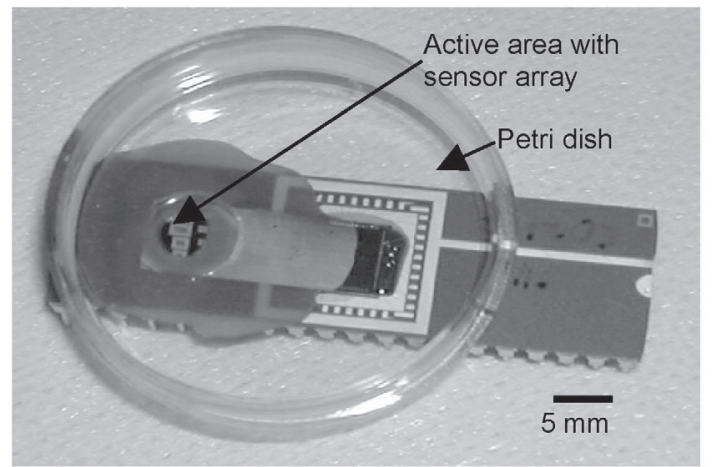

Figure 2. Fabricated sensor array, integrated into a petri dish and bonded to a DIP package, ready for use. Cells are cultured on the active area with sensor array.

As for the cell culture procedures, HL-1 atrial myocytes were plated on fibronectin coated sensors at a density of 50,000 cells/dish and cultured in serum-containing media (Claycomb media with the addition of penicillin, streptomycin, norepinephrine, fetal bovine serum, and glutamine). After reaching confluency, spontaneous beating of the cultured tissue was visually noted and the tissue was considered ready for pharmacologic testing. A single experiment utilizing one drug was performed on the tissue cultured on the oxygen sensor each day after beating was observed, thus preventing overlap from drug effects.
Physiologically relevant doses of verapamil and triiodothyronine were chosen from average serum concentrations available in published clinical studies $[\mathbf{1 0 , 1 3}]$. Solutions of media were prepared containing the following drugs at the respective concentrations: triiodothyronine at $2.5 \mu \mathrm{g} / \mathrm{L}\left(3.1 \times 10^{-4} \mathrm{mM}\right)$, and $25 \mu \mathrm{g} / \mathrm{L}\left(3.1 \times 10^{-5} \mathrm{mM}\right)$ and verapamil at $2.5\left(5.5 \times 10^{-6} \mathrm{mM}\right), 25$ $\left(5.5 \times 10^{-5} \mathrm{mM}\right)$, and $100 \mu \mathrm{g} / \mathrm{L}\left(2.2 \times 10^{-4} \mathrm{mM}\right)$. Control solution (placebos) consisted of Claycomb media [7] without drug added but prepared in the same manner as the other drug solutions (i.e., mixed and heated in a water bath). Solutions were heated to $37^{\circ} \mathrm{C}$ just before entering the chamber and intra-chamber temperatures were monitored and maintained at $36 \pm 1^{\circ} \mathrm{C}$. Voltammetric curves with excitation voltages varying from $-0.2 \mathrm{~V}$ to $-0.8 \mathrm{~V}$ were recorded at 30 second intervals for a total of 240 time points. Unless otherwise noted, experiments were run using the following procedure: after perfusing the culture for $20 \mathrm{~min}$ and obtaining a baseline, the low concentration of the drug (or placebo) was perfused for $30 \mathrm{~min}$, the first washout phase Claycomb media was perfused for $20 \mathrm{~min}$, and finally this same schedule was repeated for the high dose concentration and final washout. A typical response of an experimental run following this schedule is presented in Figure 3.

All recordings were performed using a potentiostat (Electrochemical Workstation CH600A, CH Instruments, Austin, Texas) connected to a laptop computer. Six experiments were performed for each of the two drugs as well as for the control group. The current value at a particular voltage for each time point was extracted from the data set for each experiment. Student's ttest was applied for each data set. Voltages were typically analyzed at $0.6 \mathrm{~V}$ or $0.7 \mathrm{~V}$, depending on the particular sensor and the range of voltages at which the current fell in the diffusiongoverned region as determined by the calibration curves.

During analysis, the current signal representing the oxygen concentration recorded during the 20 minutes prior to introduction of the drug-containing solution was used as a baseline. Subsequent measurements were evaluated as a percentage of this baseline. A sustained change in signal from baseline appearing after perfusion with the drug was considered to be a drug effect. The average signal over the time course of the drug effect was utilized as a representation of the effect of the drug on oxygen concentration during that period. An inversely proportional relationship between metabolism and oxygen concentrations at the interface between the tissue and sensor was assumed.

Besides calibration with three oxygen levels, temperature dependence of the oxygen sensor was characterized by gradually heating the perfusion media in the appropriate temperature range over the course of 30 minutes and monitoring the response of the current. Sensor response without cells showed exponential behavior with temperature increase, as predicted by the Nernst equation [12]. Other parameters investigated included signal response of the sensor to the same drugs without a cell culture overlaying the sensor and with a dead cell culture overlaying the sensor over the course of 30 minutes, the response of the sensor system to the introduction of fresh media independent of drug response every 5 minutes over 30 minutes, and the effect of vigorous mixing on the concentration of oxygen within a solution and the resulting effect from the metabolic sensor set-up.

\section{RESULTS}

Regression analysis of calibrations of the oxygen sensors produced linear fits with correlation coefficient values of 0.78 to 0.95 . These calibrations were utilized in quantifying oxygen concentration versus time during the experiments. Thyroid hormone's effects on tissue metabolism began over 30 minutes 
following initial perfusion with the drug while the effects of verapamil were seen more rapidly, typically around 10 minutes following the beginning of drug perfusion.

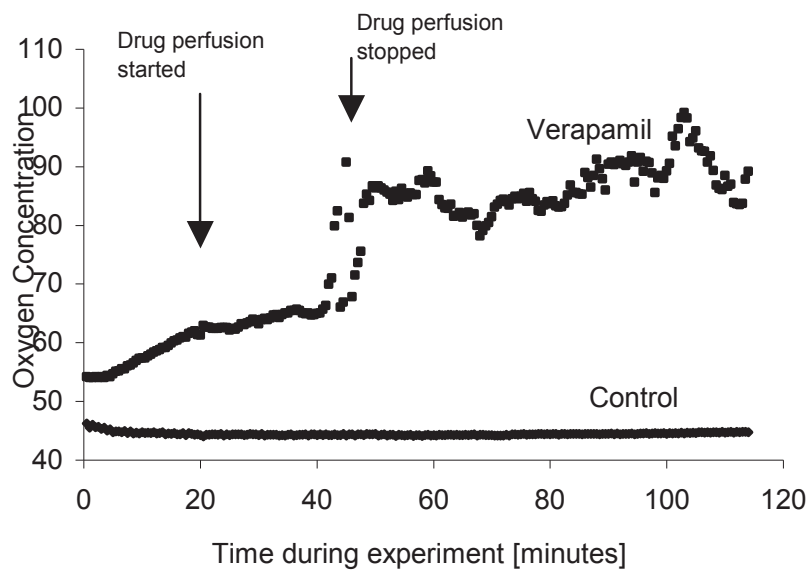

Figure 3. Example of oxygen concentration changes for control condition and under the effect of verapamil $(25 \mu \mathrm{g} / \mathrm{L})$. Either verapamil (upper curve) or placebo (bottom curve) was perfused from 20 to $50 \mathrm{~min}$, and again from 70 to $100 \mathrm{~min}$. Claycomb solution was perfused from 0 to 20, 50 to 70 , and 100 to $120 \mathrm{~min}$. The exact cause of the upward drift of the baseline oxygen concentration during the first 20 minutes has not been elucidated.

The percentage of oxygen concentration present within the cell-sensor interface during various phases of the experiment was plotted for each drug. Verapamil demonstrated a statistically significant increase in oxygen concentration compared to the control solution. As presented in Figure 3, the drug effect is clearly observed after the initial perfusion with verapamil (low dose, initiated at 20min), but after the first washout phase (from 50 to $70 \mathrm{~min}$ ) and subsequent application of the high dose (from $70 \mathrm{~min}$ on), it is not straightforward to analyze the compound effect of both drug applications. The dose dependent nature of the verapamil response could not be verified, although the decrease in metabolism by the HL-1 cells can be undoubtedly shown. For the $2.5 \mu \mathrm{g} / \mathrm{L}$ dose, the relative increase in oxygen concentration as compared to baseline was $8 \%$. At the high dose of $25 \mu \mathrm{g} / \mathrm{L}$, the increase was $35 \pm 4 \%$. A bar plot of these results is shown in Figure 4, where the data for the control experiments as well as the triiodothyronine responses are summarized.

We also tested a higher dose of verapamil, $100 \mu \mathrm{g} / \mathrm{L}$, in order to compare tissue response to the previously proposed drug administration schedule. In this case, only one dose was used, as opposed to the low and high sequential doses, but the same results were obtained. Oxygen concentration increased to $110 \pm 9 \%$ ( $\mathrm{n}=5$ experiments, 20 data points/experiment).

Thyroid hormone (T3) produced a statistically significant decrease in the oxygen concentration present at the cell sensor interface as compared to the placebo solution; although the trend of this response appeared to be dose dependent, this behavior was not proven statistically significant in these experiments. The low dose of $\mathrm{T} 3$ decreased extracellular oxygen concentration to $85 \pm 9 \%$ as compared to baseline $(98 \pm 5)$. The high dose, $25 \mu \mathrm{g} / \mathrm{L}$, produced a decrease to $77 \pm 12 \%$. These results were obtained as means and standard deviations of six independent experimental runs. Figure 4 summarizes these results.

A commercially available glucometer was utilized to confirm either increases or decreases in metabolism from the effluent stream every 10 minutes during all experiments. A summary of all experimental data is contained in Figure 5.

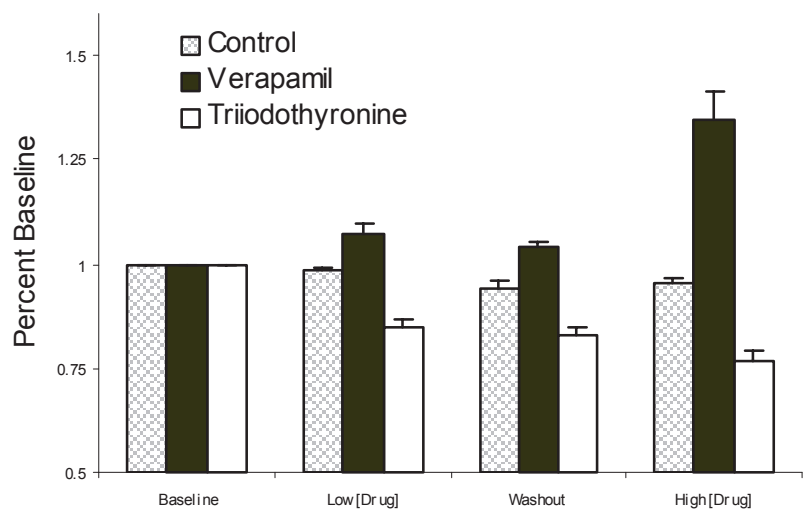

Figure 4. Relative response of oxygen concentration for control condition (placebo), verapamil and triiodo-thyronine application. The first set of columns refer to baseline (20min, $n=40$ data points/experiment), the second set refers to the applied low-dose (30min, $n=60$ data points/experiment), the third presents washout (20min), and the fourth shows the response for the high dose (30 min).

Although temperature-dependent responses were characterized with and without cells, in order to exclude any temperature-related variation, either by the electrochemical reaction or by the cellular metabolism itself, the cell culture dish was maintained at a constant temperature during data acquisition. In order to achieve temperature stability, perfusion was maintained at the same flow rate throughout the experiment. Control experiments in which no cells were used show an absence of response to drug application or solution exchange between used and new media. A 30 min period was usually required to attain sensor stability, either in experiments with or without cells, as can be observed in the initial phase of the control curve in Figure 3.

\begin{tabular}{|c|c|c|c|c|c|c|c|}
\hline & Dose & EME & OME & $\operatorname{Avg} n$ & SE (\% & Dose Dep & $\begin{array}{l}\text { Gluc } \\
\text { Verify }\end{array}$ \\
\hline \multirow[t]{2}{*}{13} & $\begin{array}{l}2.5 \mu \mathrm{g} / \mathrm{L} \\
(\mathrm{N}=6)\end{array}$ & $\uparrow$ & $(15.3 \pm 9.0 \% \mathrm{p}=0.005)$ & 44 & 4.2 & \begin{tabular}{|l|} 
Yes \\
(trend)
\end{tabular} & Yes \\
\hline & $\begin{array}{l}25 \mu \mathrm{g} / \mathrm{L} \\
(\mathrm{N}=6)\end{array}$ & $\uparrow$ & $\uparrow_{223.3 \pm 13 \% \mathrm{p}=0.007)}^{\uparrow}$ & 34 & 3.3 & $\mathrm{P}=0.12$ & Yes \\
\hline \multirow[t]{3}{*}{ VP } & $\begin{array}{l}2.5 \mu \mathrm{g} / \mathrm{L} \\
(\mathrm{N}=6)\end{array}$ & $\downarrow$ & $(7.48 \pm 10 \% \mathrm{p}=0.04)$ & 49 & 4.2 & No & Yes \\
\hline & $\begin{array}{l}25 \mu \mathrm{g} / \mathrm{L} \\
(\mathrm{N}=7)\end{array}$ & $\downarrow$ & $(34.7 \pm 4.3 \% \mathrm{p}=0.02)$ & 33 & 3.3 & & Yes \\
\hline & $\begin{array}{l}100 \mu \mathrm{g} / \mathrm{L} \\
(\mathrm{N}=5)\end{array}$ & $\downarrow$ & $\downarrow$ & - & - & & - \\
\hline
\end{tabular}

Figure 5. Effects of drugs on myocardial tissue. "T3"= triiodothyronine. "VP"=verapamil. "EME"=expected theoretical effect of drug on tissue metabolism. "OME"= effect on metabolism inferred from increase/decrease in oxygen consumption during application of drug compared to baseline. Statistical significance is calculated with comparison to the observed effect of control. "Avg n"=average number of time points at which the observed drug effect was measured in each experiment. SE=average standard error as determined by unpooled analysis of experiments expressed in percent. Dose dependency ("Dose dep") is based upon varying responses of doses representing separate means. "Gluc verify" denotes whether the observed increase/decrease in metabolism as measured by the oxygen sensor was verifiable with a standard glucose assay. "- " = analysis was not performed. 


\section{DISCUSSION AND CONCLUSION}

Dissolved gas sensors operate by electrochemically reducing oxygen dissolved in solution and measuring the resulting current. The main advantage of the presented sensor is that the electrodes are contained within an oxygen permeable PTFE membrane, which prevents signals from other electroactive species from interfering with the measurement of oxygen concentration.

The experiments described here used this advantage to measure changes in oxygen concentration at the surface of cultured myocardial tissue as the tissue was perfused with various metabolically altering drugs. Metabolic changes were inferred from this data assuming that metabolism was directly proportional to oxygen utilization and, thus, inversely proportional to oxygen concentration measured at the extracellular space.

The metabolic activity, as reflected by the concentration of oxygen at the tissue surface, was augmented by thyroid hormone (triiodothyronine, T3) which is consistent with T3's role in increasing cellular metabolism. The effects of this medication typically occurred after thirty minutes, during the washout phase of the experiment, which was expected, given its known mechanism of action.

Decreases in metabolic activity of the tissue were observed following exposure to verapamil and these effects were seen more rapidly than the metabolic effects of $\mathrm{T} 3$. These observations are consistent with both the mechanism and expected time course of action of this medication. Although it seems that the metabolic responses to drugs are dose-dependent, this could not yet be shown statistically. Factors that may have contributed to the variance in the magnitude of the drug effect include the dependence of the absolute level of metabolic activity on the location of the sensor relative to the tissue itself, the influence of oxygen availability and level of contractility on the measured drug effect.

The Nernstian behavior of the electrochemical reduction of oxygen explains part of the observed temperature dependence of the sensor responses. Temperature stability was maintained throughout the experiments in order to assure that the oxygen reduction currents in the diffusion region of measured voltammetric curves were consistent throughout experiments.

Both increases and decreases in metabolism were confirmed by changes in glucose concentration. However, the relative changes in glucose concentration were too small to be precisely measured with the device used in this study, thereby precluding any significant analysis of this data (not shown).

In conclusion, these experiments demonstrated the feasibility of monitoring metabolic changes using a recently developed Clark-type oxygen sensor. This type of measurement has many conceivable uses such as toxin detection and drug discovery. The ability to couple any type of metabolically active cell with the oxygen sensor allows for versatility. In addition, the detection of a wide variety of biologically active substances, including those that act at the cellular as well as the genetic levels, may be performed with a single type of cell because changes in cellular metabolism are a common endpoint for most drug interactions. Further sophistication in drug screening or toxin detection is possible by combining the sensitivity of such a metabolic sensor with other more specific analyzing sensors, such as those that measure the binding affinity of drugs to specific target receptors.

Though promising data was observed and statistically significant changes in metabolism were measured during the application of each of the drugs investigated, rigorous investigation of signal variability remains to be performed. This variability may occur at different levels and may involve 1) minute-to-minute variability in cellular metabolism; 2) environmental changes such as temperature drift or protein deposition; 3) inherent sensor variability. Further work will focus on quantitative approaches to correlate levels of metabolism with measured oxygen concentrations. Future experiments will make use of an independent confirmation of metabolism, such as an inline glucometer, and will focus on cells in which the use or production of an easily measured variable has a one-to-one relationship with metabolism. At that point, the resolution and repeatability of metabolic changes detectable by this oxygen sensor system may be determined.

\section{ACKNOWLEDGMENTS}

This work is funded by DARPA under contract N666001-96-C8631 and Medtronic Corporation (Minneapolis, MN, USA).

\section{REFERENCES}

1. S.A. Gray, J.K. Kusel, K.M. Shaffer. "Design and demonstration of an automated cell-based biosensor" Biosensors and Bioelectronics, 16:7-8 535-42 (2001).

2. A.M. Aravanis, B.D. DeBusschere, A.J. Chruscinski, et al. "A genetically engineered cell-based biosensor for functional classification of agents" Biosensors and Bioelectronics, 16:7-8 571-7 (2001).

3. K.H. Gilchrist, V.N. Barker, L.E. Fletcher et al. "General purpose, field-portable cell-based biosensor platform." Biosensors and Bioelectronics, 16:7-8 557-564 (2001).

4. H.K. Maerz, R. Buchholz, F. Emmrich, et al. "Applicating fiber optical methods for toxicological testing in vitro" Proceedings of SPIE - The International Society for Optical Engineering, 3603: 228-236 (1999).

5. L.C. Clark, "Monitor and Control of Blood and Tissue Oxygen Tension" Transactions of the American Society of Artificial Internal Organs, 2:144-56 (1956).

6. G.A. Langer (Ed.), The Myocardium. Academic Press, San Diego, (1997).

7. W.C. Claycomb, N.A. Lanson, B.S. Stallworth, et al, "HL-1 cells: a cardiac muscle cell line that contracts and retains phenotypic characteristics of the adult cardiomyocyte" Proceedings of the National Academy of Science, 195:2979-84 (1998).

8. A.J. Drake-Holland, MIM Noble (Ed), Cardiac Metabolism, John-Wiley and Sons. Chichester (1983).

9. W.H. Dillman. "Thyroid hormone influences on the cardiovascular system: molecular and clinical studies" Thyroid Today, 24:3-4 (2001).

10. M.J. Antonaccio (Ed.). Cardiovascular pharmacology. Raven Press, New York (1990).

11. G.W. McLaughlin, K. Braden, B. Franc, G.T.A. Kovacs, "Solid-state dissolved oxygen sensor test matrix using a pulsedplasma deposited PTFE film” Transducers '01, 2:1692-95 (2001).

12. A.J. Bard, L.R. Faulkner (Ed). Electrochemical Methods, Fundamentals and Applications. John Wiley and Sons, New York, (2001).

13. J. Morganroth, E.N. Moore (Ed). "The Evaluation of beta blocker and calcium antagonist drugs" Proceedings M. Nijhoff, Boston (1982). 